\title{
Criminologie
}

\section{La victimologie et le viol, un discours complice}

\section{Renée Collette-Carrière}

Volume 13, numéro 1, 1980

Regards sur la victime

URI : https://id.erudit.org/iderudit/017116ar

DOI : https://doi.org/10.7202/017116ar

Aller au sommaire du numéro

Éditeur(s)

Les Presses de l'Université de Montréal

ISSN

0316-0041 (imprimé)

1492-1367 (numérique)

Découvrir la revue

Citer cet article

Collette-Carrière, R. (1980). La victimologie et le viol, un discours complice.

Criminologie, 13(1), 60-79. https://doi.org/10.7202/017116ar d'utilisation que vous pouvez consulter en ligne.

https://apropos.erudit.org/fr/usagers/politique-dutilisation/ 


\title{
LA VICTIMOLOGIE ET LE VIOL, UN DISCOURS COMPLICE
}

\author{
Renée Collette-Carrière
}

Le viol! Un sujet tabou! Un mot qui fait peur! Un crime dont sont légalement victimes les femmes ! Un crime dont les auteurs sont par définition légale des hommes! Le viol! Un mot qui a une connotation péjorative et stéréotypée ! Comment arriver à cerner ce phénomène à la fois si peu connu et pourtant si «à la une »? Comment parler de la réalité non pas des mythes? Comment convaincre de la nécessité d'en parler? Comment le démystifier pour qu'enfin l'on comprenne ce dont il est vraiment question et que les hommes et les femmes aient envie qu'il disparaisse? Utopie?

Le viol! En traitant d'un sujet à la fois si d'actualité et si méconnu, il nous semble poursuivre essentiellement deux objectifs. D'abord articuler brièvement une réflexion sur le pourquoi et le comment de l'engouement suscité actuellement autour de ce crime défini légalement de manière si spécifique. Ensuite tenter de comprendre théoriquement et pratiquement les apports d'une perspective victimologique. Y a-t-il ou devrait-il y avoir une victimologie propre au viol ? Et si oui, de quelle nature est-elle présentement et que pourrait-elle être?

Pour atteindre notre premier objectif, il nous paraît opportun de situer l'état actuel du discours littéraire et scientifique et ses différents apports. D'un même souffle, nous exposerons la réalité présente du code pénal canadien. Nous vérifierons également quelques statistiques. Par la suite, nous aborderons notre second thème en scrutant d'abord ce que sont les mythes entourant le viol et nous regarderons d'un cil critique la perspective victimologique, ses différentes conséquences et les possibles apports que cette branche de la criminologie pourrait générer. Pour ce faire, nous exposerons les changements envisagés et souhaitables dans ce débat réel et actuel.

\section{Pourquoi un tel engouement?}

Une revue exhaustive de la littérature sur le viol nous permet une première constatation, soit la relative pauvreté des recherches et des écrits sur cette question avant les dix dernières 
années et plus particulièrement en ce qui concerne les victimes elles-mêmes. Il nous faut ensuite reconnaître un fait important : c'est à la montée du courant féministe actuel que nous devons les plus riches contributions. En effet, c'est aux militantes féministes américaines que revient le mérite d'avoir considéré le viol non pas seulement comme un crime à caractère sexuel mais davantage comme un acte de violence, une intrusion, une agression.

\section{Les féministes et le viol}

C'est aux États-Unis à partir de la fondation du N.O.W. (National Organization for Women) par, entre autres, Betty Freidan, en 1966, que s'amorce une critique des conceptions traditionnelles de la condition féminine. Le mouvement féministe a donc, en premier lieu, porté son attention sur la situation globale de la femme, ses droits fondamentaux pour ensuite, un peu parallèlement, se tourner vers un domaine plus restreint, sa criminalité dans ce qu'il est convenu d'appeler (peut-être à tort?) un domaine dévolu traditionnellement aux femmes : la prostitution et sa répression. L'intérêt s'est par la suite tourné vers la violence. Violence faite aux femmes! Violence des hommes! Les féministes américaines ont d'abord pris conscience de l'absence flagrante de recherches et d'écrits scientifiques à ce sujet et de la nécessité vitale de combler cette lacune. De plus, elles ont réalisé combien importantes et prises pour acquises pouvaient être certaines explications plus ou moins stéréotypées quant au viol lui-même, aux agresseurs et aux victimes. Elles ont alors avec Susan Brownmiller décidé d'organiser une vaste rencontre de discussion à ce sujet qui eut lieu à New York au début de l'année 1971. Pour Susan Brownmiller, ce fut le point de départ d'une recherche pour retracer les origines historiques du viol, sa réalité légale et judiciaire; le rôle qu'il joue dans la littérature, les journaux, le cinéma ; qui sont les victimes et les agresseurs ; comment est-il réprimé et comment devrait-il l'être? Et surtout quel est son fondement idéologique? Sa recherche dura quatre années. Elle sera publiée en anglais en 1975 et traduite en français en $1976^{1}$. Entre la rencontre et cette publication, il y aura d'importantes contributions dont Griffin (1971), Amir (1971), Weis et Borges (1973), Medea et Thompson (1974), Schwendinger et Schwendinger (1974), Mackellar (1975) et Chappell, Geis et Geis (1977).

1. Nous dressons en bibliographie une liste des principaux ouvrages. 
Plusieurs ont été traduits en français. En France, Fargier (1976) et Choisir (un mouvement de masse) (1978) ont fait le point sur cette même question. Au Canada, les contributions originales sont rares. Seules, pour le moment, Clark et Lewis (1977) nous fournissent dans un livre pertinent des données intéressantes. Il s'agit principalement d'une étude des viols commis dans la région du Toronto métropolitain.

\section{Quelques repères historiques}

C'est à Susan Brownmiller (1975) que nous devons d'avoir retracé les origines historiques du viol. Elle nous révèle que le viol a été longtemps un moyen de s'approprier une épouse. Cette méthode d'épousailles a même continué d'exister en Angleterre jusqu'au Xve siècle. Elle nous apprend en outre que le plus ancien code juridique écrit que nous connaissions, soit le code d'Hammourabi, établit certaines règles strictes par rapport à certaines catégories de femmes : par exemple pour le viol d'une vierge promise au mariage, l'agresseur devait être tué ; pour celui d'une femme mariée, l'assaillant partageait le blâme avec sa victime et tous deux étaient plongés dans les eaux du fleuve. Cependant le mari pouvait permettre que sa femme en soit retirée et le roi que l'homme soit gracié. Chez les Hébreux, le châtiment était la lapidation et il n'y avait pas de grâce possible.

L'existence juridique du viol comme crime dans le code n'est pas dù au fait que ce soit un crime contre une femme mais plutôt parce qu'il est un crime contre la propriété d'un homme soit le père soit l'époux. Dans la loi hébraïque, le viol d'une jeune vierge en dehors des murs de la cité entraînait les conséquences suivantes : l'auteur du crime dédommageait le père de la victime en lui payant une compensation monétaire pour le prix que celui-ci aurait eu au moment du mariage et il devait épouser la jeune fille. Dans le cas d'un viol d'une jeune promise, l'auteur était lapidé et la violée vendue à prix réduit à un autre homme. Pour un viol à l'intérieur des murs, l'agresseur et la victime étaient lapidés car elle aurait pu crier et être secourue donc sa culpabilité était plus grande. Après avoir ainsi retracé les origines juridiques du concept de viol, Brownmiller essaie de comprendre le viol en temps de guerre. Viols nombreux et impunis ! Armes de guerre ? Pour beaucoup, le viol est alors considéré comme un inévitable accident de parcours. Il y a toujours des viols quelle que soit la 
cause défendue. Pour la Première Guerre mondiale, Brownmiller a retrouvé des documents impressionnants sur de tels viols accompagnés de sadisme meurtrier. La Seconde Guerre nous a donné encore de multiples preuves à cet effet. Le viol a été un instrument d'humiliation du peuple vaincu par les Allemands d'abord et les alliés ensuite. Elle nous décrit l'abondance de viols au Vietnam. Il y avait là davantage qu'un accident de parcours inévitable. La prostitution était courante dans les camps américains et pourtant les viols suivis de meurtres étaient monnaie courante. Le massacre de My Lai (16-03-78) en fait foi. Il y a aussi de multiples documents mis en preuve par Brownmiller. La guerre au Bangla Desh a donné lieu à beaucoup de viols. Et ce sont les victimes qui, après la guerre, ont été le plus sévèrement pénalisées car leurs familles les ont rejetées. Il a fallu l'aide internationale pour parvenir à solutionner le problème des camps de femmes victimes de viols et sans ressources. Ce que dit Brownmiller, en étayant de sérieux documents peu ou pas connus, c'est que * la guerre foumit aux hommes la parfaite toile de fond psychologique permettant de donner libre cours à leur mépris des femmes \$ (p. 44, édition française). La guerre exalte la force virile du militaire. Elle fournit comme d'ailleurs l'esclavage, un cadre institutionnel qui confère aux assaillants un avantage certain. D'autres exemples de tels cadres sont la prison, les autorités policières et les relations d'autorité par rapport aux enfants. L'auteur nous fait donc une excellente analyse de ces types de viols. Marie-Andrée Bertrand (1979) affirme que :

Brownmiller réussit à démontrer comment ce crime est beaucoup plus que l'acte singulier d'un homme à l'endroit d'une femme. Les guerres et les occupations qu'elles entraînent, les invasions, les colonisations ont toujours et partout été l'occasion pour des hommes, vainqueurs et dominateurs, de violer les femmes des hommes vaincus et colonisés. Avec la terre et les biens - les épouses et les jeunes filles et parfois les enfants deviennent les terrains d'occupation et la nouvelle propriété des vainqueurs. Possédées par leur père, puis par leur mari, et dans quelques civilisations par leurs fils et leurs frères, les femmes sont aussi dominées par les étrangers qui occupent leur pays (p. 172).

\section{Le viol aujourd'hui}

Brownmiller s'attaque aux mythes actuels entourant le viol pour les confondre. Mais sa thèse centrale lorsqu'elle étudie le 
viol de nos jours à travers les statistiques, la littérature, les media d'information, les films, etc. peut se résumer ainsi : le viol comme crime contre les femmes constitue un moyen d'intimidation par lequel tous les hommes maintiennent toutes les femmes dans un état de peur. Elle étudie donc qui sont les victimes et quelles sont les conséquences physiques et psychologiques du viol. Elle s'aperçoit, ainsi que d'autres chercheurs (Medea et Thompson, 1974 ; Griffin, 1971 ; etc.), que toutes les femmes peuvent être violées et par l'étude des agresseurs que n'importe quel homme peut violer. Les solutions qu'elle envisage sont radicales. D'abord, elle redéfinit le viol ainsi, il s'agit :

d'une invasion sexuelle du corps par la force, une intrusion dans l'espace intérieur intime, personnel, sans consentement - en bref, une attaque intime venant d'une, entre autres, des voies d'accès et par une, entre autres, des méthodes constitue une violation délibérée de l'intégrité psychique et physique, et est un acte hostile et dégradant de violence qui mérite le nom de viol (p. 456, édition française).

Elle affirme ensuite que tous les viols devraient être rapportés et pris au sérieux. Pour ce faire, il faut que les femmes représentent la moitié des effectifs dans tout l'appareil judiciaire. Elle trouve inacceptable que la parole des plaignantes soit rejetée en cas de viol mais acceptée dans d'autres catégories de crime. Elle désire donc changer le visage des lois et de ceux qui les appliquent. Elle soutient qu'il faut punir sévèrement le violeur par une sentence de prison. Celle-ci peut varier de six (6) mois à vingt (20) ans selon la gravité de l'assaut. Elle distingue entre violence sexuelle avec ou sans arme mortelle, la violence sexuelle étant plus spécifiquement définie ainsi : « une violation de l'intégrité corporelle et une violation de la liberté et du libre arbitre quel que soit l'endroit où elle a lieu dans le lit conjugal ou en dehors $\gg$ (p. 463, édition française).

\section{Analyse féministe, la difficulté d'une politisation}

Susan Brownmiller utilise une grille d'analyse féministe mais certains auteurs y ajoutent une autre perspective, tels les Schwendinger (1974), qui utilisent un schéma marxiste. Elle analyse le viol en remettant en cause les rapports de pouvoir existant entre les hommes et les femmes. Elle les dénonce et veut provoquer un changement à deux niveaux complémentaires : d'abord 
rendre les rapports entre le féminin et le masculin égalitaires pour ensuite anéantir l'idéologie du viol.

Il paraît évident cependant que les féministes, même si elles partagent le même type d'approche au phénomène du viol, ne proposent pas toutes des solutions identiques à celle de Brownmiller. Certaines féministes, en effet, se voient prises dans une ambiguïté paradoxale. Tout en voulant un châtiment sévère pour les violeurs (affichage public de leurs noms, procès publics, prison, vendetta même...), elles reconnaissent l'inefficacité du système pénal et la nécessité de chercher des solutions non judiciaires.

\section{Le viol et la loi canadienne : le fait d'une conception patriarcale}

Au Canada, le viol est un acte criminel défini à la partie IV du Code pénal, partie qui traite des infractions sexuelles. L'article 143 stipule que :

Une personne du sexe masculin commet un viol en ayant des rapports sexuels avec une personne du sexe féminin qui n'est pas son épouse :

a) sans le consentement de cette personne du sexe féminin, ou

b) avec le consentement de cette dernière si le consentement i. est arraché par des menaces ou par la crainte de lésions corporelles; ii. est obtenu en se faisant passer pour son époux, ou jii. est obtenu par de fausses et frauduleuses représentations sur la nature et le caractère de l'acte.

La peine prévue pour le viol va jusqu'à la prison à perpétuité (article 144) et dans le cas d'une tentative jusqu'à dix (10) ans (article 145). Aux dires de ces articles, «les rapports sexuels sont complets s'il y a pénétration au moindre degré même sans émission de semence » (paragraphe 3 (6) du code criminel). Voilà donc le cadre légal qui prévaut au Canada. Les cas de viols dénoncés (puisqu'il s'agit de ceux-là) sont régis par cette loi et par une jurisprudence qui en découle. La pratique judiciaire qui s'appuie sur une législation considérant la femme victime et l'homme agresseur dénote une conception patriarcale des rapports entre l'homme et la femme. En obligeant la femme à prouver hors de tout doute raisonnable le manque de consentement, la résistance, la victime se voit souvent discréditée par les questions sur sa vie sexuelle antérieure, son statut civil, ses habitudes de vie, sa connaissance ou non de l'agresseur. Elle doit se défendre elle! C'est en effet, sur la jurisprudence des siècles passés que s'appuie 
notre pratique judiciaire actuelle. Plus particulièrement au chapitre de la crédibilité de la victime, l'arrêt Blackstone en Angleterre démontre bien la voie à suivre et suivie sans conteste. Il y est dit :

si elle (la victime) a mauvaise réputation et n'est pas soutenue par d'autres, ... si elle a tenu secret le préjudice subi pendant un temps considérable après avoir eu l'occasion de se plaindre, si l'endroit où l'acte censément commis était situé de façon telle qu'on eût pu l'entendre, et si elle n'a pas poussé de cris, pareilles circonstances font peser de fortes présomptions, fortes mais non décisives, que son témoignage peut être faux ou inventé (p. 41, dans l'édition française de Brownmiller).

En outre, une femme ne peut pas être violée par son époux ${ }^{2}$. Cette disposition de la loi canadienne semble conférer un droit de propriété au mari sur son épouse. Cette conception découle, bien sûr, de l'histoire et plus spécifiquement des ordonnances de Westminster édictées en Angleterre au XIII ${ }^{e}$ siècle. Une première ordonnance étendit la répression du viol non plus seulement aux femmes vierges mais également aux femmes mariées. La coutume de rédemption par le mariage fut abolie. De plus, la Couronne ou l'Etat eut le droit de poursuite dans ces cas. Les peines encourues alors étaient de deux (2) ans de prison ainsi qu'une amende fixée par le roi. Après dix (10) ans de cette nouvelle législation, un autre amendement fixa la peine suivante : la mort. On sanctionna cependant le droit de propriété du mari en établissant le principe selon lequel il ne pouvait avoir crime de viol au sein du mariage. Pourquoi ? Parce que le consentement de la femme au désir du mari est acquis de façon permanente par l'acte légal du mariage.

\section{Le viol : chiffre noir et statistiques}

Le viol est un des crimes le moins rapporté aux autorités policières. Et ce, même si le taux de dénonciations au Canada, par exemple, ne cesse d'augmenter depuis une vingtaine d'années. Il est difficile d'évaluer l'ampleur réelle de cette augmentation. Est-ce le nombre de viols dénoncés qui grimpe ou le chiffre réel des viols? Le chiffre noir reste toujours difficile à évaluer. Le Federal Bureau of Investigation (F.B.I.) américain estimait ces dernières années à un (1) sur six (6) le nombre de dénonciations aux Etats-Unis. Certains ont même prétendu que le taux pourrait être

2. Il y a actuellement quelques endroits qui ont enlevé l'exception mais il s'avere difficile d'obtenir justice devant les tribunaux. 
de un (1) sur vingt (20). A Montréal, les proportions de un (1) sur six (6) et parfois de un (1) sur dix (10) ont été avancées. Un fait est certain : de nombreuses victimes hésitent et refusent de porter plainte. Pourquoi? Plusieurs raisons peuvent être invoquées. Parce que la victime a peur de ne pas être crue, d'être ridiculisée ou de subir des représailles. Parce qu'elle connaissait son agresseur. Parce qu'elle a peur de l'attitude culpabilisante possible de la police, de la cour, de sa famille, de ses amis, de la société en général. Parce qu'elle se sent coupable : qu'a-t-elle bien pu faire pour que ça lui arrive à elle ? Il faut une forte dose de courage pour faire face à tous les aléas du processus judiciaire lorsque l'on sait par exemple qu'aux Etats-Unis, très peu de violeurs sont arrêtés et que $10 \%$ seulement de ces derniers sont finalement condamnés. Lorsque l'on sait aussi qu'au Québec par exemple, il existe une loi d'indemnisation aux victimes d'actes criminels (L.I.V.A.C.) depuis mars 1972 et que très peu de victimes de viols en ont bénéficié ${ }^{3}$.

Consultant les statistiques criminelles de 1971 au Canada, on constate que 2107 viols ont été rapportés et un peu plus de la moitié soit 1230 ont été déclarés fondés *. De ce nombre, il y a eu 717 arrestations $(58,3 \%)$ et seulement 119 suspects ont été inculpés $(16,1 \%)$. De ceux qui ont été inculpés, $9,1 \%$ furent condamnés, pourcentage qui ressemble beaucoup au taux américain précédemment cité.

Au Québec, les statistiques de la criminalité nous apprennent qu'en 1977, sur 480 viols rapportés et jugés fondés, 198 hommes ont été accusés ${ }^{5}$, soit $41,2 \%$. Vu la durée des procédures, il est malheureusement impossible d'établir le taux de condamnations de cette année-là. On peut se référer, cependant, à l'étude de Clark et Lewis (1977). Cette recherche démontre que, pour le Toronto métropolitain en 1970, il y a eu 116 viols dénoncés à la police impliquant 117 plaignantes et 129 agresseurs. Ceux-ci se répar-

3. L.I.V.A.C. est appliqué par la Commission des accidents du travail et peut dédommager dans les cas de lésions corporelles, grossesse, choc mental ou nerveux et décès si la demande est faite dans un délai d'un an. En 1973, la Commission a versé $\$ 5883,34$ pour sept cas d'attentats à la pudeur et de viols (soit des montants variant entre $\$ 180$ et $\$ 2500$ ) dont une pension alimentaire pour un enfant né à cause d'un viol. L'année précédente, elle avait eu une ou deux demandes (Renseignement pris dans Lord, 1977). Canada, 1971.

4. Statistiques de la criminalité, Bureau fédéral de la statistique,

5. Commission de police de Québec, Statistiques de la criminalité au Québec, no 78044. 
tissaient ainsi : 108 cas de viols impliquant un assaillant ; 6 cas de viols impliquant chacun deux agresseurs ; 2 cas de viols en groupe impliquant trois attaquants dans le premier et six dans le second.

Dans $7,4 \%$ des cas, il y avait donc plus d'un agresseur ${ }^{6}$. Sur les 116 viols rapportés, 42 furent jugés par la police comme étant fondés $(36,2 \%)$ et 74 comme étant non fondés $(63,8 \%)$. Les raisons invoquées pour décider du bien-fondé de la plainte sont : les possibilités réelles de procéder en cour; la crédibilité et la respectabilité de la victime; les marques physiques évidentes d'attaques ; les chances de retrouver l'assaillant à partir des détails fournis par la victime. Le refus de cette dernière de poursuivre les démarches par peur de représailles, par pression de sa famille ou de ses amis, par peur de la publicité et des sarcasmes des différents intervenants sont au nombre des raisons aboutissant au classement de la plainte. Clark et Lewis ont effectué leur propre classification :

Groupe A : les 42 cas déclarés fondés par les policiers ;

Groupe B : les 62 cas déclarés non fondés pour les raisons énumérées plus haut mais qui ont effectivement été des viols au sens de la loi;

Groupe $\mathbf{C}$ : les 12 cas effectivement non fondés selon toute apparence dont 4 s'étaient révélés des accusations portées par vengeance. Ces 12 cas représentent $10,3 \%$ du total (116).

À la suite de cette classification, Clark et Lewis ont utilisé pour leur recherche les 104 cas qu'elles jugèrent fondés. Les auteurs font remarquer qu'il s'agit uniquement de viols simples. En effet, les cas de viols suivis de meurtres sont rangés parmi les meurtres. De même, les cas auxquels on a donné une autre qualification, telle que attentat à la pudeur, sont difficilement retraçables. Il y a donc statistiquement des viols invisibles. Dans les cas jugés fondés par la police, on a arrêté 32 individus et la cour en a condamné 17 soit $51,2 \%$. Par rapport au nombre de plaintes officielles (116), le taux de condamnation est de $16,4 \%$ (pourcentage légèrement supérieur à celui du Canada en 1971 et au pourcentage américain). Par contre, si on estime hypothétique-

6. Tous ces chiffres proviennent du chapitre 2 de leur livre. 
ment que le nombre de viols rapportés n'est que le cinquième des viols commis, on réalise très vite que très peu de coupables sont poursuivis pour un crime aussi rebutant. Il est également facile de constater que notre système judiciaire et celui des autres pays, si on en juge par les écrits existants, pénalisent fortement la victime tout en s'avérant fort cléments à l'égard de l'agresseur. Ceci est confirmé par le taux de condamnation qui pour le viol est moins élevé que pour plusieurs des autres crimes (soit au Canada 54,6\% contre $86 \%$ pour l'année 1971). Cette clémence s'observe également dans les sentences imposées aux soixante-cinq condamnés pour viols en $1971^{\top}$ :

Type de sentence

Nombre de condamnés

\begin{tabular}{lr} 
sentence suspendue & 1 \\
moins de 2 ans & 12 \\
2 à 5 ans & 23 \\
plus de 5 ans à 14 ans & 28 \\
plus de 14 ans & $\frac{1}{65}$ \\
total & \\
\hline
\end{tabular}

Au Canada, la peine moyenne pour le viol en 1971 est donc de 4 à 5 ans de pénitencier. Or, le maximum prévu par l'article 144 du Code criminel est, rappellons-le : l'emprisonnement à perpétuité. Loin de nous la pensée d'exiger des sentences plus lourdes car il faudrait à tout le moins scruter la qualité des programmes offerts à ces criminels en prison; cependant de là à conclure qu'au Canada, le crime de viol est sévèrement réprimé, il $y$ a une marge qui relève presque du mythe.

\section{Les mythes autour du viol, du violeur et de la victime}

Ces stéréotypes qui entourent le viol, quels sont-ils ? Reprenons brièvement les plus courants :

$1^{\circ}$ Le viol est un acte spontané de la part d'un homme qui ressent un désir sexuel pressant et incontrôlable qu'il doit satisfaire immédiatement. Ce stéréotype semble se contredire par le fait que la grande majorité des viols sont prémédités. Ainsi Amir (1971) lorsqu'il étudia les viols signalés à la police de Philadelphie

7. Chiffres tirés des Statistiques de la criminalité, Bureau fédéral de la statistique, Canada, 1971. 
de 1958 à 1960, a découvert que $71 \%$ de l'ensemble avaient été planifiés dans le sens que le lieu du viol avait été prévu ainsi qu'un plan pour y amener la victime. De plus, $11,4 \%$ avaient été l'objet d'une préméditation partielle après la rencontre de la victime potentielle, tandis que $15,9 \%$ n'avaient pas été planifiés. En divisant les viols selon le nombre d'agresseurs, Amir constata que $90 \%$ des viols en groupe ont été prémédités; comparé à $82,8 \%$ des viols comprenant deux attaquants, et $58,4 \%$ dans les cas de viols à un seul agresseur. Dans la plupart des cas, il ne s'agit donc pas d'un acte spontané.

$2^{\circ}$ Le violeur est un solitaire : la même étude d'Amir (1971) démontre que $43 \%$ de viols sont commis à deux assaillants ou en groupe. Une proportion semblable a été relevée en France.

$3^{\circ}$ Le violeur est un inconnu : dans une thèse inédite sur 230 actes de violence sexuelle à Montréal, Roussel (1975) a découvert que $68 \%$ des viols ont lieu lors de rapports sociaux banaux. Amir (1971) affirme que dans $48 \%$ des cas de son échantillon, victimes et agresseurs se connaissaient. De plus, il ne s'agit ici que des cas rapportés et le fait de connaître son agresseur implique souvent que le viol ne sera jamais signalé.

$4^{\circ}$ Le violeur est un malade mental : Amir (1971) a découvert que parmi les violeurs $3 \%$ seulement avaient des troubles de cet ordre. Brownmiller (1975) rapporte des faits significatifs à ce propos. Medea et Thompson (1974) étudient les motivations des agresseurs. Elles découvrent deux types : le premier cherche la soumission totale des femmes qu'il méprise pour prouver sa virilité en niant toute manifestation féminine; pour ceci faire, il pousse à l'extrême des qualités dites viriles soient l'agressivité, la brutalité et la violence et il agresse la femme pour se prouver qu'il est un homme. Le second type de violeur est persuadé que toutes les femmes ne sont que des objets sexuels et que c'est à lui d'amener la femme à avoir des relations sexuelles indépendamment des circonstances, il n'éprouve aucun sentiment de culpabilité puisqu'il n'a pas l'impression de commettre un viol. Il saisit l'occasion, c'est tout, et c'est même son rôle!

$5^{\circ}$ Le viol est un moyen d'obtenir une satisfaction sexuelle : selon Carole Smart (1976) la violence utilisée lors du crime infirme cet avancé, car s'il s'agit seulement d'une satisfaction 
sexuelle, il n'y a pas de raison d'y mettre autant d'agressivité. Amir (1971) démontre, en effet, qu'il y a violence dans $85,1 \%$ des cas. À moins d'accepter l'hypothèse voulant que le désir sexuel ne puisse être satisfait qu'avec une démonstration de violence, il faut admettre qu'il s'agit d'un acte d'hostilité et non pas d'une simple activité sexuelle.

$6^{\circ}$ Le viol n'arrive qu'aux femmes provocantes : ceci semble être nié par le fait que les victimes sont de tout âge et de toutes apparences physiques. Parfois, elles sont même de sexe masculin.

$7^{\circ}$ Toutes les femmes désirent être violées et quand elles disent non, elles pensent oui : c'est sous-entendu le concept freudien du mascchisme féminin. Le viol est l'archétype de l'expérience de femme (Hélène Deutsch). Toutes les femmes savent qu'un acte sexuel non désiré est atroce, surtout si cet acte est accompagné de violences physiques ou intimidantes. Pourquoi ne se défend-elle pas davantage? Par peur! Elle est littéralement paralysée. Conditionnée depuis sa plus tendre enfance à avoir peur, à être passive. Elle a peur de cet homme qui est censé être un protecteur. Il y a là une sorte de cadre d'autorité subtil fortement intégré par la socialisation. Les effets de cette socialisation conditionnent les modes de réaction et les moyens utilisés pour se défendre contre une attaque. Dans notre culture, la femme ne s'attend pas à être attaquée et n'a pas appris à se défendre.

\section{La victimologie, ou l'absence d'un discours novateur}

La socialisation en perpétuant l'apprentissage des rôles selon un modèle passif-féminin, actif-masculin, contribue à rendre la femme "victimisable ». La victimisation, processus social par lequel un individu devient victime, donc vulnérable, paraît être le lot des femmes. L'homme n'est pas conditionné à ce rôle de victime ; il se doit d'être fort, agressif, protecteur. C'est donc toute l'idéologie de la sexualité qui est ici en cause, idéologie selon laquelle la femme attend que l'homme la séduise. Or. cette séduction peut devenir forcée, c'est un viol. Malgré ce fait, elle apparaît aux yeux de certains presque légitime. Du même souffle, on attend d'une femme qu'elle soit passive, douce, affectueuse (pas trop), attentive aux efforts masculins pour la séduire et si elle se plaint d'une attaque, on exige alors qu'elle prouve sa résistance et son non-consentement. Le fardeau de la preuve lui incombe et, 
ne l'oublions pas, le viol est le seul délit ainsi libellé. Qu'elle a appris à ne pas se défendre contre une attaque sexuelle ou non, elle est immobilisée par la peur et par sa faiblesse physique et psychologique de se protéger elle-même. Weis et Borges (1973) soulèvent cette question en expliquant que la première réaction de la victime c'est d'abord une négation de ce qui arrive ; et plus longue cette période durera, plus longue sera la période d'inaction de sa part, et plus grand sera l'avantage psychologique et physique de l'attaquant.

L'apport de la victimologie à l'étude du viol ne semble pas avoir été très positif. La victimologie, comme le terme l'indique, est l'étude des victimes, de leur rôle dans le déroulement du crime, dans la prévention du dit crime et/ou dans la résolution du conflit.

Les écrits en victimologie se sont attardés principalement au rôle de la victime dans le cas du viol et en ce faisant ils ont renforcé les mythes entourant ce crime. En tentant de rechercher une part de responsabilité même inconsciente du côté de la victime, cette branche de la criminologie a perpétué le schéma existant de discréditation de la victime, un schéma sous-entendant entre autres choses que cette dernière a pu ressentir du plaisir lors du viol. Carole Smart (1976) soutient qu'en maintenant une telle croyance, on en fait ainsi un crime sans victime. On finit par donner encore moins de droit aux victimes. On déplace le blâme du violeur vers la violée. D'ailleurs, Clark et Lewis (1977) définissent ainsi la victimologie : l'art de blâmer la victime. Et, en effet, elle peut facilement devenir une forme de rationnalisation pour expliquer un comportement criminel. En rejetant le blâme sur la victime, le viol est presque excusé, justifié. Or, c'est déjà toute la réalité du processus judiciaire dans les cas de violeurs poursuivis. En discréditant la victime, la défense tente de prouver que celle-ci l'avait bien cherché ou mérité, que laccusé n'est pas vraiment coupable. Les questions sur la vie sexuelle antérieure de la victime ${ }^{8}$ n'ont pour but que de démontrer à la cour que la victime ayant eu une vie sexuelle antérieure a pu fort bien consentir au dit viol ! Pourtant, il n'y a là aucun lien direct sinon des

8. Malgré qu'au Canada, le Bill C-71 adopté en 1976, stipule que le huis-clos puisse être demandé et que de telles questions soient soumises par écrit au juge qui pourrait les accepter ou non, on sait que cette procédure n'est pas toujours respectée. 
préjugés d'ordre moral. L'exemple du viol d'une prostituée est intéressant à ce chapitre. Elle peut avoir effectivement été sauvagement violée, mais elle n'est pas une victime « respectable», elle n'est pas une femme digne et elle ne sera pas crue. D'ailleurs Clark et Lewis (1977) dans leur étude démontrent que d'autres types de femmes sont «inviolables ». Elles le deviennent par le système de filtrage, sélection effectuée par la police qui reflète la conception de la femme que véhicule la société. Parmi ces victimes ou ces femmes inviolables est la femme qui a bu ou qui est droguée, la jeune fille qui ne vit plus chez ses parents et qui est considérée comme ayant un comportement difficile, la femme dont l'âge se situe entre 30 et 40 ans et qui est soit séparée, divorcée ou qui vit en concubinage. La chômeuse ou la femme qui reçoit des prestations du bien-être social. La femme qui est sous les soins d'un psychiatre. Celle qui n'a pas rapporté le crime à la première personne rencontrée après sa commission. Celle qui connaissait son attaquant. Celle qui a accepté d'aller à sa résidence ou de faire un tour d'auto. "Les femmes qui présentent certains de ces facteurs ne verront pas leur assault sexuel défini comme un viol. Elles ne peuvent littéralement pas être violées parce que le viol est défini légalement et socialement de telle sorte qu'elles ne sont pas le genre de femme que la société croit pouvoir être violée» (p. 92, notre traduction). Ce sont donc des femmes qui ont une position dépréciée dans notre société.

La libération sexuelle a amené un affrontement entre les mœurs anciennes qui distinguaient nettement entre les femmes respectables et les femmes «faciles», et les mœurs nouvelles selon lesquelles les femmes veulent être libres sexuellement et décider pour elles-mêmes, libres également de vivre seule, de voyager seule, de marcher seule sur la rue. En s'écartant de la «respectabilité traditionnelle * ces femmes sont devenues des proies. Medea et Thompson (1971) tentent ainsi d'expliquer l'augmentation du nombre de viols. Elles disent:

C'est pourquoi de nos jours, le violeur prend le meilleur du monde ancien et du nouveau : il lui tombe sous la main des femmes qui prennent plus de risques, tandis que la société continue d'affirmer que si elles prennent de tels risques elles finissent par avoir ce qu'elles méritent (p. 59, édition française).

Le viol n'est pas en règle générale, le fait d'individus ayant des troubles mentaux spécifiques, nous l'avons dit antérieurement. 
Il est donc souvent l'acte d'un homme tout à fait ordinaire mentalement normal. Or, en victimologie, ces constatations nous paraissent, au moins jusqu'à un certain point, se retourner contre la victime. On tombe dans un autre piège : si l'attaquant est un homme normal c'est peut-être qu'il est poussé à commettre un tel assaut sexuel parce que la victime l'y a incité.

Amir (1971) tombe dans un type d'explication parvenant à blâmer certaines victimes. Il faut lui reconnaître le mérite d'avoir effectué et publié la première étude statistique d'envergure sur le viol. Il n'a toutefois pas cherché à étudier les viols étiquetés non fondés. Il a aussi introduit le concept sociologique de victim precipitation ou viction precipitated rape que nous traduisons par * action précipitante de la part de la victime . Il en parle dans le cas \& de situations de viol au cours desquelles la victime, en réalité ou il en fut induit ainsi, a accepté d'avoir des relations sexuelles mais se ravisa avant l'acte ou n'a pas réagi avec conviction au moment de la suggestion du délinquant * (p. 266, notre traduction) ${ }^{8}$. Il s'agit donc de l'interprétation des actions de l'un par l'autre. Amir pense que c'est à la victime d'assumer la responsabilité des mauvaises interprétations de ses propres actions car tout comportement contraire aux attentes sociales d'une femme respectable peut entraîner une telle mésinterprétation. Il dit que cela est vrai pour $19 \%$ des cas de son échantillon. Pour Clark et Lewis (1977) ce concept n'est pas défini clairement. Il se réfère à des situations « chargées sexuellement » pouvant entraîner un viol. Certains éléments en sont la mauvaise réputation de la victime, la présence d'alcool, la rencontre dans un bar ou lors d'une réception. Il s'ensuit que la femme se trouve toujours dans une impasse : si elle adopte une attitude passive, cela pourrait être interprété comme un assentiment. Si elle adopte un comportement actif, cela serait vu comme une promesse. Rendre la victime responsable des interprétations d'un partenaire masculin, c'est encore culpabiliser la victime. Chappell, Geis et Geis (1977) rappellent qu'Amir a puisé ce concept chez Wolfgang lorsqu'il traite de l'homicide. Ce dernier parle du comportement contributoire mais de façon très spécifique et claire. Il s'agit souvent de cas où victimes et meurtriers avaient des antécédents judiciaires

9. Rape situations in which the victim actually, or so it was deemed, agreed to sexual relations but retracted before the actual act or did not react strongly enough when the suggestion was made by the offender. 
similaires et où l'étincelle entraînant le meurtre fut allumée par la victime. Pour Chappell, Geis et Geis (1977) c'est davantage d'un état de vulnérabilité qu'il faudrait parler. Par exemple : faire du pouce est apte à rendre la fille hautement vulnérable. Ils ajoutent que dans le cas d'un viol, il est très rare que ce soit la victime qui incite le violeur.

\section{En guise de critique}

Les explications victimologiques de cette nature se caractérisent par l'absence de remise en question des fondements idéologiques et culturels que sous-entendent les définitions des rôles sexuels dans notre société. C'est ainsi que l'on continue de perpétuer les mythes même si on prétend les remettre en question. En prenant pour acquis la définition traditionnelle des rôles sexuels, on accepte et on excuse la sexualité agressive chez l'homme tout en consacrant et sanctionnant la vulnérabilité de la femme. On peut bien sûr parler, d'état de vulnérabilité, de degré de risque plus ou moins élevé selon les situations, mais on oublie que celles-ci ne sont pas nécessairement voulues par les femmes. Par exemple, une infirmière qui travaille le soir ou une employée qui termine à minuit et doit passer devant un champ vacant pour rentrer chez elle, sont davantage vulnérables mais doivent-elles changer de travail ? Golda Meir dans une discussion sur le sujet au cours de laquelle un de ses ministres proposait l'imposition du couvre-feu pour les femmes répondit : « Mais ce sont les hommes qui attaquent les femmes. Si on doit imposer le couvre-feu, que les hommes restent à la maison, non pas les femmes. »

\section{Et les solutions}

Il semble y avoir un certain consensus quant à la nécessité d'apporter des solutions et des changements. Il y a cependant, divergence quant à la nature de ces changements. Quant à nous, les solutions nous paraissent se situer à trois niveaux. D'abord sur le plan légal, ensuite au niveau de situations concrètes de viol et finalement par rapport à une éducation possible, afin de changer les mentalités.

\section{Le viol et la loi}

Certains changements sont présentement proposés par différents intervenants. Dans un récent article écrit en collaboration 
avec Me Céline Lamontagne, nous avons exposé ces différentes propositions et $\mathrm{Me}$ Lamontagne a pris position ${ }^{10}$. Sans reprendre en détail ces propositions, il semble opportun d'en préciser deux points : d'abord en ce qui concerne le projet de loi C-52 déposé en première lecture en mai 1978, mais pas encore adopté. De plus, au cours d'une récente déclaration radiophonique, l'ancien ministre de la Justice, Me Marc Lalonde, a annoncé son intention d'y apporter l'amendement suivant : au lieu de transformer le viol en attentat à la pudeur simple ou grave, il y aurait deux crimes : soit ingérence sexuelle (sentence maximale : 5 ans) et agression sexuelle (sentence maximale : 10 ans). En outre, la Commission de réforme du droit du Canada (CRD) dans un rapport ( $\left.\mathrm{n}^{\circ} 10\right)$ continue à privilégier un changement d'appellation et une mise en évidence du caractère agressif de l'acte tel que nous le décrivions à partir de son document de travail ( $\mathrm{n}^{\circ}$ 22). Cependant, la Commission a changé ses recommandations initiales. Arrêtonsnous à la description de ses nouvelles propositions : que les articles 143-144-145-149 et 156 (attentat à la pudeur) et 157 (grossière indécence) soient abrogés et remplacés par les deux articles suivants :

1. attouchement sexuel : "quiconque, dans un but sexuel, touche directement ou indirectement une autre personne sans le consentement de cette dernière est coupable : a) d'un acte criminel et passible d'un emprisonnement de 5 ans; ou b) d'une infraction punissable sur déclaration sommaire de culpabilité »(p. 17).

2. agression sexuelle : \&quiconque emploie ou menace d'employer la violence dans la perpétration d'un attouchement sexuel est coupable d'un acte criminel et passible d'un emprisonnement de 10 ans $\gg$ (p. 17).

La Commission recommande que l'exception retenue pour les maris qui violent leurs femmes soit supprimée. Il va donc plus loin que dans son premier document, mais tout en maintenant toutefois la nécessité de prouver le non-consentement ce qui, à notre avis, continue d'imposer aux victimes le fardeau de la preuve.

Certains changements sont aussi proposés par les groupes féministes dont le Conseil du statut de la femme (CSF) du Québec

10. Voir $\mathbf{R}$. Collette-Carrière et $\mathrm{C}$. Lamontagne, $\&$ Le viol au $\mathrm{Ca}-$ nada : un débat renouvelé», Déviance et Société, Genève, Eđitions médecine et hygiène, Vol. 3 , no 1,1979 , p. 83-88. 
dans son rapport «Égalité et Indépendance 》 dont nous résumons la teneur dans l'article cité. Le Conseil canadien du statut de la femme (CCSF) ainsi que d'autres groupes ont aussi avancé des propositions visant la réforme de la loi actuelle.

Nous croyons que la loi ne devrait pas contenir des préoccupations d'ordre moral. L'appellation de viol doit disparaître. L'aspect sexuel de l'agression ne doit pas être privilégié car cet acte est avant tout une atteinte à l'intégrité d'un individu quel qu'il soit.

\section{Le viol, les centres et l'information}

Quel type d'aide concrète devons-nous apporter aux victimes de violence sexuelle ? Il existe des centres fort valables, capables d'intervenir dans ces situations de crise. Ils sont d'un grand secours pour la victime et ce, sur tous les plans. Ils ont prouvé leur efficacité. Malheureusement, il nous faut déplorer la fermeture de tels centres à Montréal. Manque de ressources financières et possiblement mésententes quant à la vocation de ces centres, voilà les raisons ${ }^{11}$. Doit-on se consacrer à l'information et l'éducation du public au sujet du viol ou fournir de l'aide à une clientèle spécifique : les victimes? Ne peut-on laisser à d'autres organisations féminines le soin de s'en occuper ou même aux hôpitaux directement? Il nous semble que les centres devraient exister et remplir ces deux fonctions. D'une part accueillir et aider les victimes et d'autre part éduquer et informer le public pour que le sujet ne soit plus tabou ainsi que faire le lien avec les services policiers, hospitaliers et judiciaires.

\section{Changer les mentalités}

Un troisième niveau d'action est celui des changements de mentalité. Et c'est là, nous semble-t-il, que la victimologie a un rôle positif à jouer. Il faut faire un choix : continuer de chercher la faute du côté de la victime ou redonner à l'auteur du viol la responsabilité de son acte. La victimologie doit aller au-delà des stéréotypes et des explications traditionnelles. Elle doit étudier les effets réels du viol sur les victimes, les vraies attitudes du violeur, pour pouvoir modifier les mécanismes idéologiques qui régissent les rapports sociaux entre les deux sexes.

11. Article du journal la Presse du 4 mai 1979, p. D-2. 
Cesser de perpétuer la dichotomie qui existe au niveau des rôles sexuels, permettra à l'homme et à la femme, bien que différents, d'être tout simplement des humains et des égaux. Ils ne seront plus dans un rapport supérieur-inférieure, actif-passive ou dominant-dominée. La victimologie comme la criminologie en général peut faire porter le débat sur les causes inhérentes au crime de viol. Elle peut contribuer à la recherche des vraies dimensions du phénomène. Elle doit travailler à la prévention de ce crime, être créatrice à ce niveau et ainsi aider à la résolution de cet agir criminel.

Il ne faut plus considérer le viol comme un fait naturel et inéluctable. Nous sommes face à un fait social, résultat d'un certain type de rapports entre les hommes et les femmes. Ces rapports sont issus, Marie-Andrée Bertrand en parle avec clarté dans son livre la Femme et le crime, d'une conception basée sur l'hégémonie normative de la société soutenue par les appareils idéologiques d'état. Pour bloquer cette réalité, pour ne plus perpétuer le viol, il faut cesser de croire que les femmes sont inexorablement des victimes et les hommes inéluctablement des agresseurs.

\section{BIBLIOGRAPHIE}

AMIR, Menachem, 1971 : Patterns in Forcible Rape, Chicago, University of Chicago Press.

BERTRAND, Marie-Andrée, 1979 : La Femme et le crime, Montréal, Collection Exploration, L'Aurore/Éditions l'Univers.

BROWNMILLER, Susan, 1975 : Against our will :Men, Women and Rape, New York, Simon and Schuster. En français : 1976, Le Viol, ParisMontréal, Stock-Éditions l'Étincelle.

CHAPPELL, Duncan, Robley GEIS, Gilbert GEIS, editors, 1977 : Forcible Rape, the crime, the victim and the offender, New York, Columbia University Press.

CHOISIR (un mouvement de masse), 1978 : Viol, le procès d'Aix-enProvence, Paris, Editions Gallimard.

CLARK, Lorenne, Debra LEWIS, 1977 : Rape : The price of coercive sexuality, Toronto, Women's Educational Press.

CODE CRIMINEL CANADIEN, 1978 : Montréal, Wilson et Lafleur Limitée.

COLLETTE-CARRIÈRE, Renée, Céline LAMONTAGNE, 1979 : Le viol au Canada : un débat renouvelé ", Déviance et société, Genève, Éditions médecine et hygiène, Vol. 3 , no 1 , p. 83-88.

Note : dans ce numéro, il y a trois autres articles sur le même sujet et l'ensemble constitue le débat sur le viol et la justice pénale. 
COMMISSION DE RÉFORME DU DROIT DU CANADA, 1978 : Infractions sexuelles, Droit pénal, Document de travail no 22, Ottawa, Ministère des Approvisionnements et Services Canada.

COMMISSION DE REFORME DU DROIT DU CANADA, 1978 : Rapport sur les infractions sexuelles, no 10, Ottawa, Ministère des Approvisionnements et Services Canada.

CONSEIL DU STATUT DE LA FEMME, 1978 : Pour les Québécoises : égalité et indépendance, Québec, Éditeur Officiel du Québec.

FARGIER, Marie-Odile, 1976 : Le Viol, enquête, Montréal et Paris, Éditions l'Étincelle et Éditions Grasset et Fasquelle.

GRIFFIN, Susan, 1971 : \& Rape : The All-American Crime >, Ramparts Magazine, no 10. En français, 1971 : Le Viol, Montréal, Éditions l'Étincelle.

LORD, Catherine, 1977 : \&Les femmes en ont ras le viol \$, Revue Châtelaine, Montréal, Juin 1977.

MACDONALD, John M., 1971 : Rape Offenders and their Victims, Springfield, Charles C. Thomas.

MACKELLAR, Jean, 1975 : Rape, the Bait and the Trap, New York, Crown Publishers. En français, 1975 : Le Viol, l'appat et le piège, Paris, Edition Petite Bibliothèque Payot.

MEDEA, Andrea, Kathleen THOMPSON, 1974 : Against Rape, New York, Farrar, Straus and Giroux. En français, 1976 : Contre le viol, Paris, Pierre Horay éditeur.

PROJET DE LOI C-52, présenté à la Chambre des Communes du Canada, $3^{\text {e }}$ session, $30^{\circ}$ Législature en mai 1978, Information Canada.

ROUSSEL, Gilles, 1975 : Délits contre la personne et délits sexuels d Montréal, Thèse de maîtrise inédite, Centre de documentation, Université de Montréal.

SCHWENDINGER, Julia R., Herman SCHWENDINGER, 1974 : Rape Myths : In Legal, Theoretical and Everyday Practice, Crime and Social Justice, a Journal of Radical Criminology, Spring-Summer, no 1 , p. 18-27.

SMART, Carol, 1976 : Women, Crime and Criminology, a Feminist Critique, London, Routledge et Kegan Paul.

STATISTIQUES DE LA CRIMINALITÉ, 1971 : Division de la statistique judiciaire, Ottawa, Bureau fédéral de la statistique, Canada.

STATISTIQUES DE LA CRIMINALITE AU QUÉBEC, 1977 : Commission de police de Québec, Québec, no 78044.

WEIS, Kurt, Sandra S. BORGES, 1973 : Victimology and rape : the case of the legitimate victim, Issues in Criminology, School of Criminology, Berkeley, Fall, vol. 8, no 2. 